\title{
Influence of Cross-Linker Concentration on Physical Properties of Main-Chain Liquid Crystalline Elastomers
}

\author{
Supardi ${ }^{a, b}$, Harsojo $^{a}$, Yusril Yusuf ${ }^{a *}$ \\ ${ }^{a}$ Physics Department, Universitas Gadjah Mada, Sekip Utara BLS 21, 55281, Yogyakarta, Indonesia \\ ${ }^{b}$ Physics Education Department, Universitas Negeri Yogyakarta, Karangmalang, 55281, Yogyakarta, \\ Indonesia
}

Received: April 13, 2017; Revised: July 12, 2017; Accepted: July 27, 2017

\begin{abstract}
We studied physical properties of main-chain liquid crystalline elastomers (MCLCEs) to observe the influence of variations of cross-linker concentrations on properties such as spontaneous deformation, elastic free energy, and order parameter. Cross-linker concentrations of four MCLCE samples were $8 \%, 12 \%, 14 \%$, and $16 \%$. Samples with higher cross-linker concentrations underwent spontaneous deformation to a greater extent than those with lower concentrations, indicated by a smaller aspect ratio of length from the end to the beginning of heating. We also reformulated the elastic free energy expression to observe the dependence of the physical quantity on temperature variation. It showed that the maximum relative elastic free energy of the MCLCEs occurred at different temperatures, dependent on cross-linking density, where higher density caused the maximum relative free energy to decrease. The cross-linker concentration also significantly influenced the order parameter of the samples, where the higher the cross-linker concentration, the higher the maximum order parameter.
\end{abstract}

Keywords: elastic free energy, main-chain liquid crystalline elastomers, order parameter, spontaneous deformation.

\section{Introduction}

Liquid crystalline elastomers (LCEs) are materials that combine two unique properties, namely, an anisotropic property carried by mesogenic moieties and elasticity of the conventional cross-linked polymer networks ${ }^{1-4}$. The anisotropic nature is generated by the mesogenic units within the LCEs reorganizing themselves so their orientational direction is preferentially aligned with a particular direction called director $\hat{\mathbf{n}}^{5-7}$. They have an orientational order but still have the capability to flow with respect to one another ${ }^{8}$. A nematic-isotropic phase transition can be generated by heating, applying an electric or magnetic field or adding non mesogenic solvent ${ }^{3,8,9}$. Imposing an external stimulus, such as heating, causes LCEs to contract or expand in alignment with or in a perpendicular direction to the director $\hat{\mathbf{n}}^{10}$. Such contraction or expansion can be quite large and reversible ${ }^{3,8}$, so LCEs have potential in technological applications such as actuators $^{11-15}$, sensors ${ }^{14}$, and artificial muscles ${ }^{11,16-20}$.

There are two types of liquid crystalline polymers (LCPs), namely, main-chain LCPs (MCLCPs) and side-chain LCPs (SCLCPs), depending on where the mesogenic moieties are attached to the polymer backbone. Schematics of the MCLCPs and SCLCPs are shown in Figure 1 (a) and Figure 1 (b and c), respectively. In the MCLCPs, the pendant rods are incorporated into the polymer backbone, whereas in the SCLCPs, they are attached to the backbone polymer via flexible spacers that can be side-on or end-on. The LCPs, both main-chain and side-chain, can be covalently cross-linked to form a three-dimensional network called LCEs. Due to the coupling of the rod-like segments to the polymer backbone, for the same degree of nematic ordering, the MCLCEs will stretch to a greater extent than the SCLCEs. The monodomain of these systems can be expanded up to $400 \%$ compared to the SCLCEs ${ }^{18}$. This impressive property provides the potential for MCLCEs as actuators and artificial muscles ${ }^{8,20-22}$.

Efforts are being devoted to obtaining more promising MCLCEs, although fabrication is more complicated ${ }^{10,21}$. Aguilera and Ringsdorf ${ }^{22}$ succeeded in synthesizing MCLCP with the mesogenic units based on three aromatic rings and oligosiloxane as the chain extender. Donnio et al. ${ }^{23}$ synthesized the first MCLCE by cross-linking the LCP with a flexible siloxane-based cross-linker. Rousseau et al. ${ }^{24}$ synthesized anew silicon-based main-chain LCP with the tunable clearing temperature controlled by chemical composition. Bispo et al. ${ }^{25}$ synthesized an MCLCE with various approaches to obtain MCLCE with a nematic phase in the range of room temperature and systematically reduce the formation of a smectic mesophase. By using a spin casting technique, Sanchez-Ferrer and Finkelmann ${ }^{21}$ synthesized a new MCLCE with a monomer that was vinyloxy-terminated and 


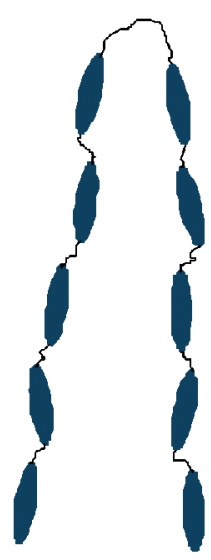

(a)

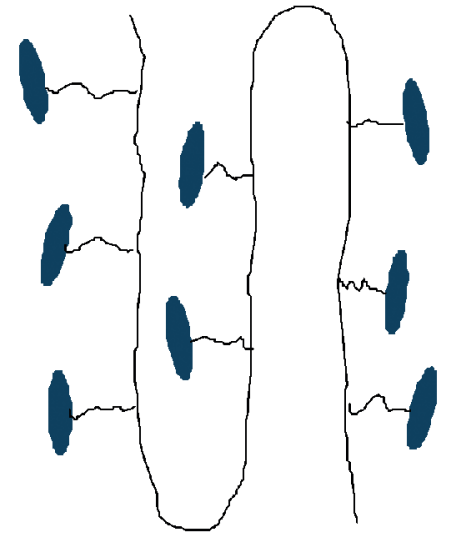

(b)

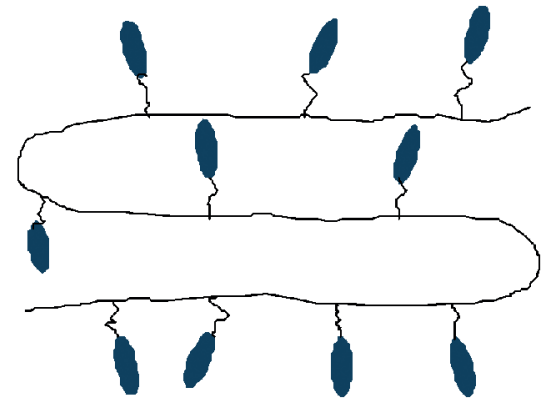

(c)

Figure 1. Schematic picture of MCLCPs (a), side-on SCLCPs (b), and end-on SCLCPs (c) ${ }^{3}$.

a pentamethylcyclopentasiloxane-based cross-linker linked directly to the mesogenic units in the polymer backbone. The mechanical and thermal actuation properties of MCLCE materials have also been studied ${ }^{3,10,14,23,25}$ and show the exponential behavior of strain close to the clearing temperature $\left(T_{\mathrm{c}}\right)$. Some factors influence the mechanical properties, namely, the nature of mesogenic units, the degree of cross-linking, and the connectivity of the mesogenic units to the polymer backbone (side chain or main chain) ${ }^{8}$. Chemically, there is a relationship between the cross-linker constitution and its mechanical effects ${ }^{25}$ and between the LC polymer chain architecture and its anisotropy ${ }^{3}$.

This paper presents the results of an experimental study conducted to reveal the influence of the cross-linking density and temperature on the physical properties of spontaneous deformation, elastic free energy, and order parameter, of MCLCEs with various cross-linker concentrations. The spontaneous deformation was obtained by comparing the sample length in the nematic and isotropic phases. The results provide a description of spontaneous shrinkage when the samples were heated from $30^{\circ} \mathrm{C}$ to $110^{\circ} \mathrm{C}$. Calculation of the elastic free energy was based on a special expression given by Warner and Terentjev ${ }^{3}$, with a little modification and assumption. Based on the spontaneous deformation data, we analyzed the order parameter changes during a heating and fit them to a suitable function to find the relationship between the cross-linking density and the maximum order parameter.

\section{Experimental Procedure}

We prepared four MCLCE samples with various crosslinker concentrations: 8\% (MCLCE-8), 12\% (MCLCE-12), 14\% (MCLCE-14), and 16\% (MCLCE-16). These specimens were synthesized by Krause et al. ${ }^{26}$ by a one-step reaction, dissolving the 2-ethyl-1, 4-phenylene bis [4- [4- (vinyloxy) buboxy] benzoate] $\left(\mathrm{C}_{34} \mathrm{H}_{38} \mathrm{O}_{6}\right)$ monomer, the chain extender of 1,1,3,3,-tetramethyldisiloxane $\left(\mathrm{C}_{4} \mathrm{H}_{14} \mathrm{OSi}_{2}\right)$, and $8 \mathrm{~mol}-\%, 12 \mathrm{~mol} \% \%, 14 \mathrm{~mol}-\%$, and $16 \mathrm{~mol}-\%$ of the pentamethylcyclopentasiloxane $\left(\mathrm{C}_{5} \mathrm{H}_{20} \mathrm{O}_{5} \mathrm{Si}_{5}\right)$ cross-linking agent in toluene. The clearing temperature for the samples were $\sim 90{ }^{\circ} \mathrm{C}$ (MCLCE-8), $\sim 99^{\circ} \mathrm{C}$ (MCLCE-12), $\sim 103{ }^{\circ} \mathrm{C}$ (MCLCE-14), and $\sim 90^{\circ} \mathrm{C}$ (MCLCE-16) ${ }^{9}$. The monodomain MCLCEs were obtained by mechanical stretching to obtain the uniform director orientation, which was aligned to the stretching direction.

We heated the samples in a hot stage at a temperature ranging from $30{ }^{\circ} \mathrm{C}$ through $110^{\circ} \mathrm{C}$. The shape changes were observed using a polarizing microscope with a charged coupled device (CCD) camera connected directly to its ocular lens, and a personal computer (PC) was applied to record any shape changes during a heating. Figure 2 shows the experimental device set-up to observe the spontaneous shape change as a function of temperature; the device consists of a polarizing microscope, CCD camera, temperature controller, multimeter, and PC. A glass containing the specimen was placed on the hot stage, which had a heater inside.

\section{Results and Discussion}

\subsection{Spontaneous deformation}

A spontaneous deformation, either a contraction or expansion, will occur when external stimuli such as temperature or a magnetic or electric field are imposed on LCEs. In the case of heating, when the temperature increases, the contraction will take place in alignment with the director $\hat{\mathbf{n}}$, and at the same time, expansion will occur in the direction perpendicular to the director $\hat{\mathbf{n}}$. Despite the shape change due to the external stimuli, the volume of the MCLCEs remains unchanged. 


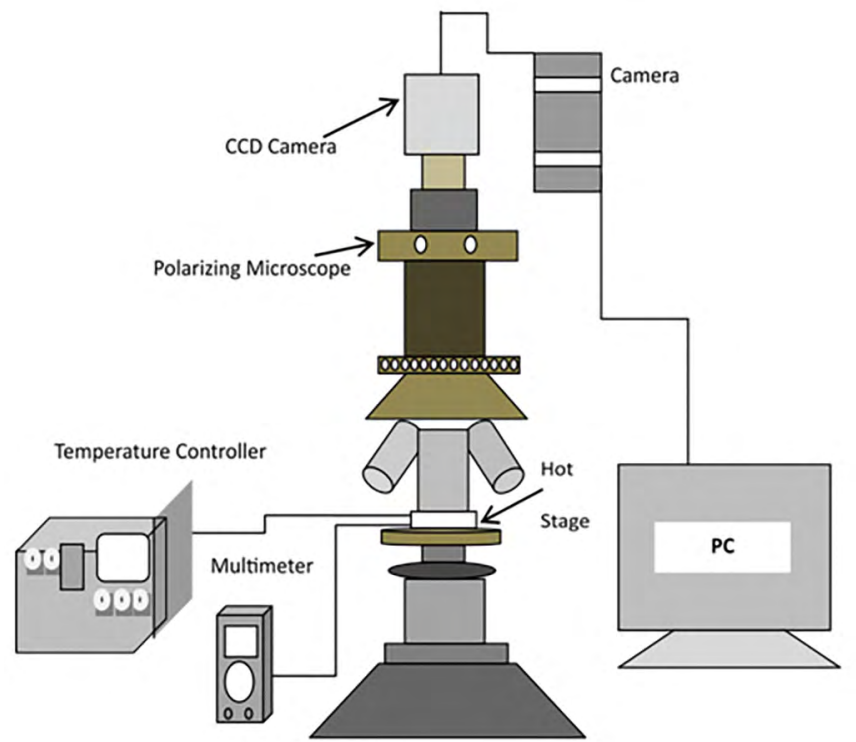

(a)

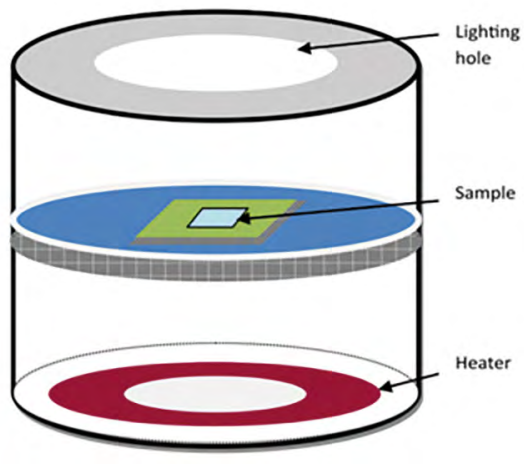

(b)

Figure 2. Experimental set-up to observe the spontaneous shape change as a function of temperature; the device consisted of a polarizing microscope, CCD camera, temperature controller, multimeter, hot stage, and PC (a). A glass containing the specimen was placed on the hot stage $(b)^{27}$.

Figure 3 shows the relationship of the relative length change as a function of temperature when the four MCLCE specimens were heated from $30^{\circ} \mathrm{C}$ to $110^{\circ} \mathrm{C}$. For MCLCE-8 and MCLCE-12, the graphs show almost flat at the start of heating, at a temperature of about $30^{\circ} \mathrm{C}$ to $40^{\circ} \mathrm{C}$. This indicates that the samples have already undergone contraction and expansion at ambient temperature, although the expansion is relatively small. The contrast occurs with MCLCE-14 and MCLCE-16, which have started to change shape from the beginning of heating. A drastic change also occurs at a temperature of about $60^{\circ} \mathrm{C}$ to $70{ }^{\circ} \mathrm{C}$ for MCLCE-8 and MCLCE- 16 and at about $75^{\circ} \mathrm{C}$ to $85^{\circ} \mathrm{C}$ for MCLCE- 12 and MCLCE-14. The drastic change happens when the system approaches the samples' clearing temperature. MCLCE-8 and MCLCE-16 experience spontaneous deformation earlier than MCLCE-12 and MCLCE-14, since these samples' clearing temperatures are lower than those of MCLCE-12 and MCLCE-14. After the samples enter the isotropic phase, they do not experience any deformation, so the relative length change remains constant.

Figure 3 also shows that the highest cross-linking density specimen (MCLCE-16) deforms earlier than others, whereas the lowest cross-linking density sample (MCLCE-8) is practically unchanged at the beginning of heating. This may be explained as follows. At the time of MCLCE formation, the ordering level of the mesogenic units is relatively the same. Since the LCPs are cross-linked with various cross-linking densities, the degree of crystallinity of the MCLCE samples with lower cross-linking density becomes higher than that of the samples with higher density, as confirmed by the previous study ${ }^{28}$.

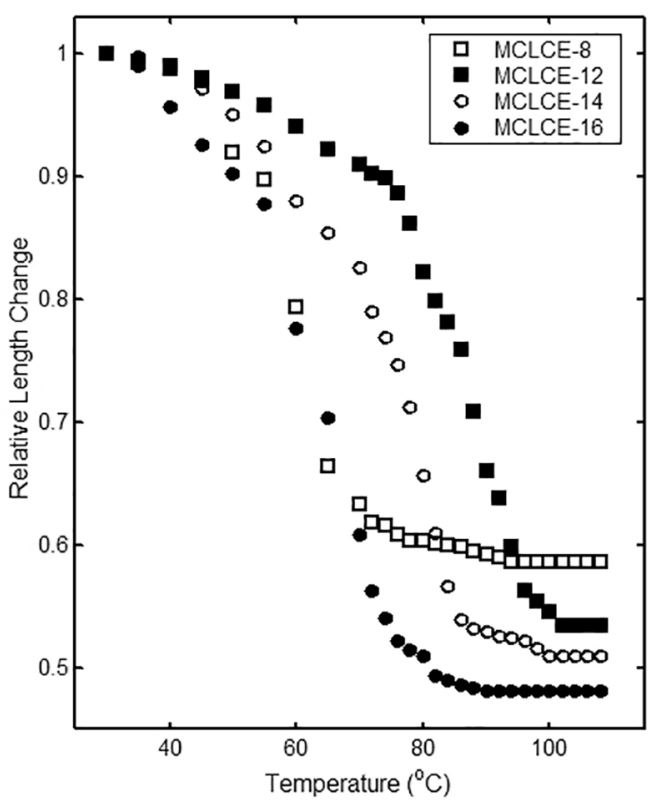

Figure 3. Relative length change along the director $\hat{\mathbf{n}} l(\mathrm{~T}) / l(30$ ${ }^{\circ} \mathrm{C}$ ) as a function of temperature for MCLCE-8, MCLCE-12, MCLCE-14, and MCLCE-16. All the samples undergo a drastic change in length in the vicinity of the clearing temperature and no change when entering their isotropic phase.

At the beginning of heating, a slight change in samples with higher cross-linker concentrations will soon be detected. In contrast, the MCLCE samples with lower cross-linking densities practically do not change in shape, because their degree of crystallinity is higher than that of the others, resulting in a slight undetectable shape change of the material. 
Figure 4 displays a linear regression of the maximum aspect ratio change with respect to the cross-linker concentration when the samples contract at the final heating $\left(110^{\circ} \mathrm{C}\right)$ as compared to the beginning $\left(30^{\circ} \mathrm{C}\right)$, or $\operatorname{AR}\left(110^{\circ} \mathrm{C}\right) / \mathrm{AR}(30$ $\left.{ }^{\circ} \mathrm{C}\right)$. We can see that all the graphs show as almost linear. In the final heating, the remaining lengths of MCLCE-8, MCLCE-12, MCLCE-14, and MCLCE-16, respectively, are $\sim 59 \%, \sim 54 \%, \sim 51 \%$, and $48 \%$ of the total $100 \%$ length. This means that in alignment with the director $\hat{\mathbf{n}}$, the MCLCEs with higher cross-linker concentrations contract to a greater extent than do the lower ones; this is indicated by the final length of MCLCE-16, which is the shortest among the samples. Hence, the maximum aspect ratio decreases as the cross-linking density increases. This phenomenon arises due to the effect of the cross-linker agent in the MCLCEs, which cross-link between the LCPs. When the cross-linker concentration is higher, it will better accommodate the mesogenic units in organizing themselves than when their concentration is lower, so that they are maximally able to contract.

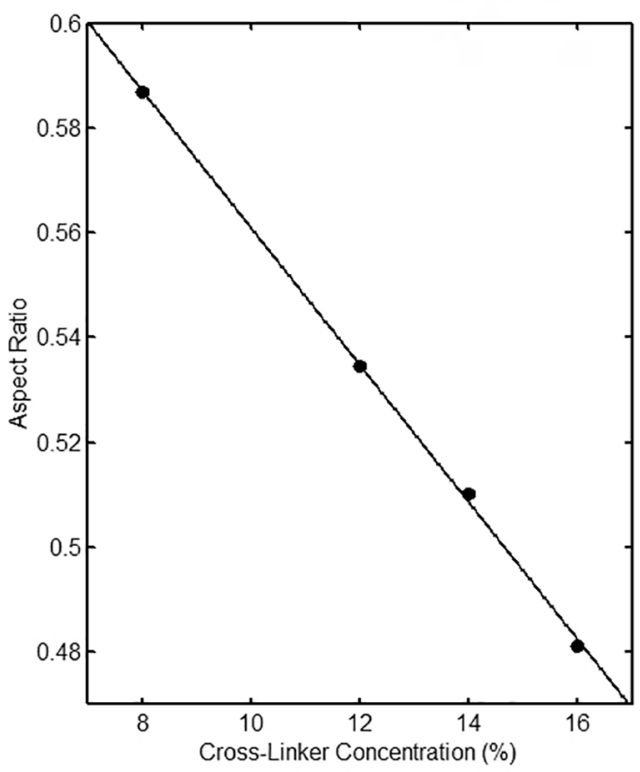

Figure 4. A linear regression of the maximum aspect ratio AR(110 $\left.{ }^{\circ} \mathrm{C}\right) / \operatorname{AR}\left(30^{\circ} \mathrm{C}\right)$ with respect to the cross-linker concentrations, where the higher the cross-linker concentration, the lower the aspect ratio.

\subsection{Elastic free energy of $L C E$}

Warner and Terentjev ${ }^{3}$ introduced a model called neoclassical elastic theory to study the elastic behavior of liquid crystalline elastomers. However, the model is not applicable, due to the step length variable depending on the direction, so that the distribution of Gaussian probability also depends on the direction. Warner and Terentjev ${ }^{3}$ have provided an expression of elastic free energy for main-chain LCEs to obtain elastic free energy at a certain temperature. However, it is impossible to measure the elastic free energy with such limited equipment. Therefore, we reformulate the elastic free energy expression so that it takes the following form:

$$
F^{L C E}=\frac{3 \mu}{2}\left(\frac{a}{l_{\|}}\right) \lambda_{m}^{2}
$$

where $\mu$ : elasticity modulus, $a$ : step length when the networks are formed in the isotropic state, $l_{\|}$: effective step length in aligning with the director $\hat{\mathbf{n}}$, and $\lambda_{m}$ : spontaneous strain. Here, we compare the elastic free energy value of each sample with respect to a standard sample to get the relative elastic free energy. We choose the elastic free energy of MCLCE-8 as a standard, since it has the lowest cross-linker concentration among the samples. In fact, samples with cross-linker concentrations lower than that of MCLCE-8 would no longer be in an elastomer shape but would be in a gel. In this case, we assume that the comparison of $\left(a / l_{\|}\right)$ between the three samples and MCLCE- 8 is the same, so the only factor that dominates is the square of spontaneous strain $\left(\lambda^{2}{ }_{m}\right)$.

Figure 5 displays the graph of the relative elastic free energy as a function of temperature. The elastic free energy of MCLCE-12, MCLCE-14, and MCLCE-16 relative to MCLCE-8 is identified, respectively, by the graphs of MCLCE8-12, MCLCE8-14, and MCLCE8-16. We notice that at the beginning of heating, MCLCE8-16 has the greatest relative elastic free energy, followed by MCLCE8-14 and MCLCE8-12, but MCLCE8-12 rises more quickly, followed by MCLCE8-14 and MCLCE8-16. The maximum elastic free energies for MCLCE8-12, MCLCE8-14, and MCLCE8-16 are, respectively, about 2.63 (at $\left.\sim 76^{\circ} \mathrm{C}\right), 2.19\left(\sim 70{ }^{\circ} \mathrm{C}\right)$, and 1.82 $\left(\sim 65^{\circ} \mathrm{C}\right)$. The peak points of the relative elastic free energy move to the left as the cross-linker concentration increases. After reaching the maximum value, the relative elastic free energies decrease drastically to achieve the isotropic state. The graph also shows that the maximum relative elastic free energies tend to decrease as the cross-linker concentration increases. This may be related to the level of difficulty in the system in developing spontaneous deformation. MCLCE-16, the sample with the highest cross-linker concentration, most easily experiences spontaneous deformation, due to having the lowest elastic free energy. The cross-linking high density of MCLCE-16 provides better memory in returning to its original shape. Thus, the other samples have more difficulty in changing spontaneously, since their elastic free energy is larger than that of MCLCE-16.

\subsection{Order parameter}

A physical quantity, called the order parameter $(Q)$, is used to describe the ordering level of a system that has a value of zero in the isotropic phase and nonzero in the anisotropic phase. In the case of MCLCEs, the order parameter is used to specify the ordering level of the mesogenic units in the 


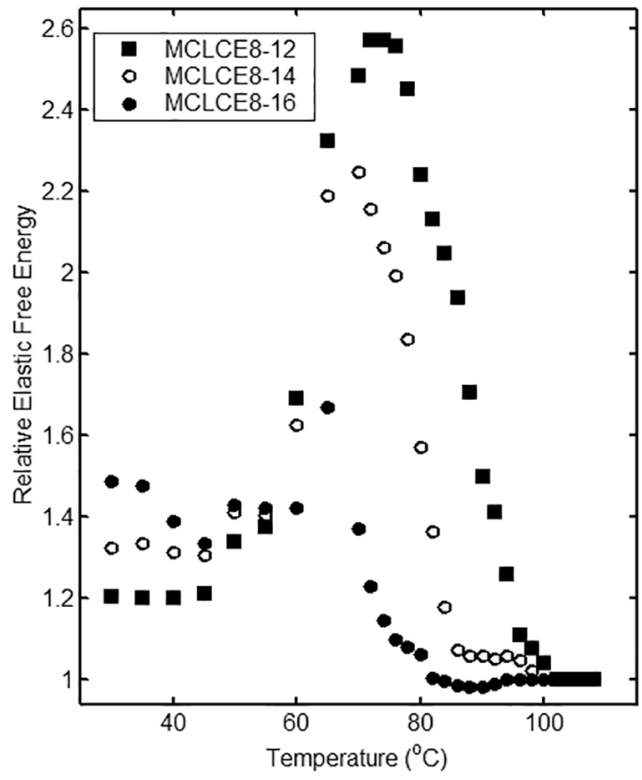

Figure 5. The graph of relative elastic free energy as a function of temperature for three MCLCE samples, with MCLCE-8 as a standard sample. MCLCE8-12, MCLCE8-14, and MCLCE8-16 are the names used to identify the relative elastic free energy of MCLCE-12, MCLCE-14, and MCLCE-16 relative to MCLCE-8. MCLCE8-12 has the highest relative elastic free energy, followed by MCLCE8-14 and MCLCE8-16. The relative elastic free energy will be the same after entering the isotropic phase.

MCLCE that point to the director $\hat{\mathbf{n}}$. Warner and Terentjev ${ }^{3}$ provided a simple expression to describe the order parameter as a function of step length ratio, expressed as follows:

$$
Q=\frac{r-1}{r+2}
$$

where $Q$ : order parameter, $r=l_{\|} / l_{\perp}=\lambda^{2}{ }_{m}$ : step length ratio, $l_{\|}$: effective step length in alignment with the director $\hat{\mathbf{n}}, l_{\perp}$ : effective step length in the perpendicular direction to the director $\hat{\mathbf{n}}$. Based on Eqn. (2), we see that the order parameter $Q$ will be equal to zero when $r$ is equal to 1 , which means that the comparison of $l_{\|}$and $l_{\perp}$ must be the same. In other words, the system no longer undergoes a shape change at all. This condition is achieved when the system enters the isotropic phase.

Figure 6 shows a variation of the order parameter during heating from $30{ }^{\circ} \mathrm{C}$ to $110^{\circ} \mathrm{C}$. We notice that the order parameter $Q$ has a value of zero to nonzero, but it is never equal to one. A zero value of $Q$ corresponds to an isotropic phase once the systems are heated, passing through their clearing temperature so that the comparisons of $l_{\|}$and $l_{\perp}$ are the same. Meanwhile, the nonzero value of $Q$ corresponds to a condition where the system is in the nematic phase, as indicated by the step length ratio $r>1$. As illustrated in the figure, the maximum value of $Q$ for all samples is achieved at the beginning of heating, namely, a state in which the system is at room temperature, and then it decreases continuously until it reaches zero. According to Eqn. (2), the $Q$ value will never reach the value of one until it is satisfied with a condition of $l_{\|} \gg l_{\perp}$, that is, a condition where the rod-like segments point in one direction only. A drastic change in $Q$ happens at a certain temperature interval, but it never discontinues. None of $Q$ shows a first-order transition. This may be due to the presence of the cross-links between the liquid crystalline polymers, which will be a constraint on the system, so that the mesogenic units in the polymer backbone do not suddenly change orientation but, rather, moderate. As a consequence, the transition phase of nematic-isotropic is no longer first-order but is closer to the second order.

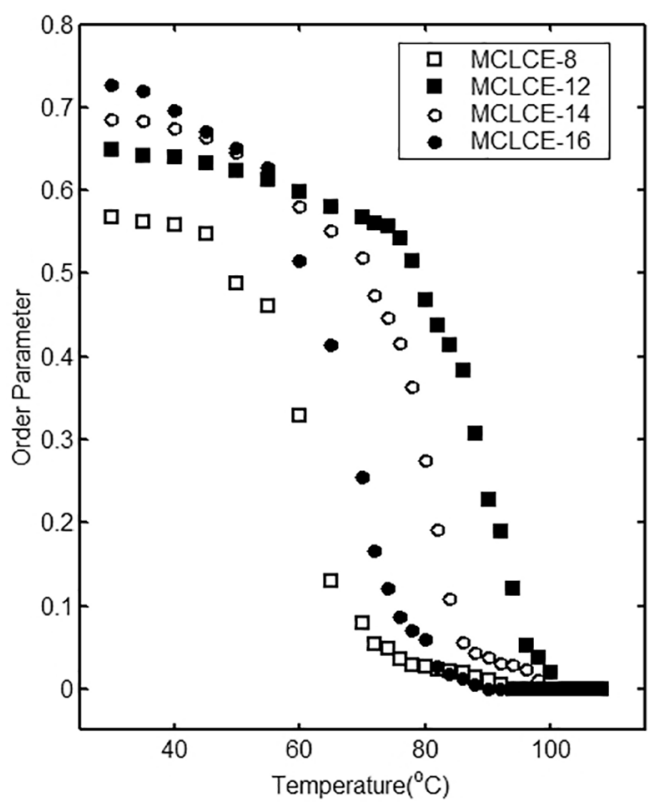

Figure 6. Order parameter dependence temperatures of MCLCEs. Each graph shows the same pattern of changing slowly at the beginning of heating and then reducing dramatically at a particular interval until the isotropic phase is found.

Figure 7 displays a linear regression that shows a description of the relationship of the maximum order parameter with respect to the cross-linker concentration. The figure shows the close relation between them, which is that when the cross-linker concentration increases, the maximum order parameter does as well. This may be due to the fact that the mesogenic units attached to the polymer backbone have a random direction or do not have an inclination in a particular direction in the isotropic phase. When the temperature is lowered so that the systems go into the nematic phase, the mesogenic units will be more ordered, as they are preferentially aligned to the director $\hat{\mathbf{n}}$. They have the opportunity to organize themselves in a preferred direction. The sample with the highest cross-linker concentration (MCLCE-16) stretches to a greater extent than do those with lower concentrations, 
as the mesogenic units that reorient to align in the stretching direction are more ordered than in the others. This condition could not be separated from the role of the cross-linker agent, which cross-links between the LCPs so they are not spilled. In this case, the higher cross-linker concentration provides support for strong bonds between the LCPs.

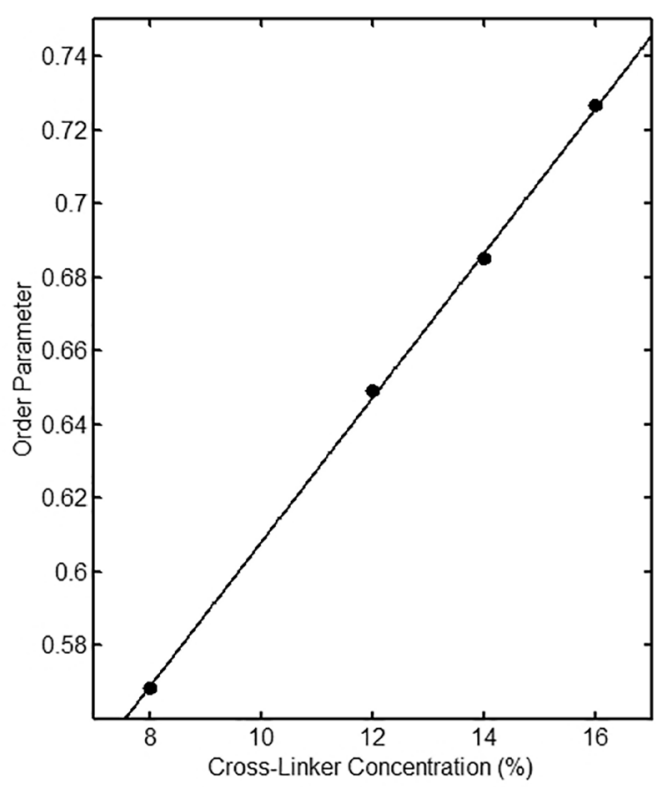

Figure 7. A linear regression of the maximum order parameter with respect to the cross-linker concentration, which shows that the higher cross-linker concentration has a higher maximum order parameter.

\section{Conclusion}

Based on the experimental results, we find that the cross-linker concentration and physical properties are closely related. Increasing the cross-linker concentration has a significant effect on the physical properties, adding the ability of the MCLCEs to deform to a greater extent; this leads to a greater shape change in alignment with the director $\hat{\mathbf{n}}$ than is seen with lower concentrations, and it decreases the relative maximum free energy. Higher concentration MCLCEs deform more easily or stretch to a greater extent in the vicinity of the nematic-isotropic phase transition temperature, which also increases the ordering level of the mesogenic units.

\section{Acknowledgements}

The authors express immense gratitude to the Indonesian Ministry of Research, Technology, and Higher Education, through PUPT Grant No. 2375/UN1-P.III/LT/DIT-LIT/2017, for financial support in this research.

\section{References}

1. Finkelmann H, Kock HJ, Rehage G. Liquid crystalline elastomers - a new type of liquid crystalline material. Macromolecular Rapid Communications. 1981; 2(4):317-322.

2. Terentjev EM. Liquid-crystalline elastomers. Journal of Physics: Condensed Matter. 1999; 11:239-257.

3. Warner M, Terentjev EM. Liquid Crystal Elastomers. Oxford: Oxford University Press; 2003.

4. Xie P, Zhang R. Liquid crystal elastomers, networks and gels: advanced smart materials. Journal of Material Chemistry. 2005; 15(26):2529-2550.

5. Küfer J, Finkelmann H. Nematic liquid single crystal elastomers. Macromolecular Rapid Communications.1991; 12(12):717-726.

6. Tajbakhsh AR, Terentjev EM. Spontaneous thermal expansion of nematic elastomers. The European Physics Journal E.2001; 6(2):181-188.

7. Thomsen DL, Keller P, Naciri J, Pink R, Jeon H, Shenoy D, et al. Liquid crystal elastomers with mechanical properties of a muscle. Macromolecules. 2001; 34(17):5868-5875.

8. Jiang H, Li C, Huang X. Actuators based on liquid crystalline elastomer materials. Nanoscale. 2013; 5(12):5225-5240.

9. Supardi, YusufY, Harsojo. Characterization of main-chain liquid crystal elastomers by using differential scanning calorimetry (DSC) method. Advanced Materials Research . 2015; 1123:6972 .

10. Yusuf Y, Hashimoto S, Cladis PE, Brand HR, Krause S, Finkelmann $\mathrm{H}$, et al. Main chain liquid crystalline elastomers: Swelling dynamics and electromechanical effects. Molecular Crystals and Liquid Crystals. 2009; 508(1):367-379.

11. de Gennes PG, Hébert M, Kant R. Artificial muscles based on nematic gels. Macromolecular Symposia. 1997; 113(1):39-49.

12. de Gennes PG. A semi-fast artificial muscle. Comptes-Rendus de l'Academie des Sciences Series IIB Mechanics Physics Chemistry Astronomy. 1997;324(5):343-348.

13. Fischl T, Albrecht A, Wurmus H, Hoffmann M, Stubenrauch M, Sánchez-Ferrer A. Actuator materials: Liquid crystalline elastomers for microengineering. Kunststoffe. 2006; 2006(10):3034.

14. Ohm C, Brehmer M, Zentel R. Applications of liquid crystalline elastomers. In: de Jeu WH, ed. Liquid Crystal Elastomers: Materials and Applications. New York: Springer; 2012. p.49-93.

15. Petsch S, Rix R, Khatri B, Schuhladen S, Müller P, Zentel R, et al. Smart artificial muscle actuators: Liquid crystal elastomers with integrated temperature feedback. Sensors and Actuators A: Physical. 2014; 231:44-51.

16. Hébert M, Kant R, de Gennes PG. Dynamics and thermodynamics of artificial muscles based on nematic gels. Journal de Physique I. 1997; 7(7):909-919.

17. Wermter H, Finkelmann H. Liquid crystalline elastomers as artificial muscles. e-Polymers. 2001; 1(1). 
18. Shenoy DK, Thomsen DL, Srinivasan A, Keller P, Ratna BR. Carbon coated liquid crystal elastomer film for artificial muscle applications. Sensors and Actuators A: Physical. 2002; 96(23):184-188.

19. Naciri J, Srinivasan A, Jeon H, Nikolov N, Keller P, Ratna BR. Nematic elastomer fiber actuator. Macromolecules. 2003; 36(22):8499-8505.

20. Li MH, Keller P. Artificial muscles based on liquid crystal elastomers. Philosophical Transactions. Series A, Mathematical, Physical and Engineering Sciences. 2006; 364(1847):27632777.

21. Sánchez-Ferrer A, Finkelmann H. Thermal and mechanical properties of new main-chain liquid-crystalline elastomers. Molecular Crystals and Liquid Crystals. 2009; 508:348-356.

22. Aguilera C, Ringsdorf H. Thermotropic polyesters with mesogenic groups based on substituted hydroquinone units and highly flexible siloxane spacer in the main chain. Polymer Bulletin. 1984; 12(1):93-98.
23. Donnio B, Wermter H, Finkelmann H. Structure, mobility, and piezoelectricity in ferroelectric liquid crystalline elastomers. Macromolecules. 2000; 33:7724-7729.

24. Rousseau IA, Qin H, Mather PT. Tailored phase transitions via mixed-mesogen liquid crystalline polymers with silicon-based spacers. Macromolecules. 2005; 38(10):4103-4113.

25. Bispo M, Guillon D, Donnio B, Finkelmann H. Main-chain liquid crystalline elastomers: Monomer and cross-linker molecular control of the thermotropic and elastic properties. Macromolecules. 2008; 41(9):3098-3108.

26. Krause S, Zander F, Bergmann G, Brandt H, Wertmer H, Finkelmann H. Nematic main-chain elastomers: Coupling and orientational behavior. Comptes Rendus Chimie. 2009;12(1-2):85-104.

27. Supardi, Harsojo, Yusuf Y. Experimental studies of thermoinduced mechanical effects in the main-chain liquid crystal elastomers. Advanced Materials Research. 2014; 896:322-326.

28. Supardi, YusufY, Harsojo. Characterization of main-chain liquid crystal elastomers using X-ray diffraction method. In: Proceedings of the International Conference on Physics 2014; 2014 Aug 2526; Yogyakarta, Indonesia. p. 16-18. 